\title{
A STUDY ON DETECTION TECHNIQUES FOR DOWNLINK IN MULTI-CARRIER CDMA SYSTEM
}

\author{
NGUYEN NGOC TIEN ${ }^{1}$, NGUYEN VIET KINH ${ }^{2}$, SEONG RAG KIM ${ }^{1}$ \\ ${ }^{1}$ Electronic and Telecommunication Research Institute - ETRI, \\ 161 Gajeong-dong, Yuseong-gu, Daejeon, 305-350 KOREA \\ ${ }^{2}$ Hanoi National University
}

\begin{abstract}
In this paper, we consider and compare several different detection techniques for the down link of a MC-CDMA system in terms of bit error rate (BER) performance. In addition, an analysis of the linear detection approach named minimum mean square error (MMSE) multi-user detection per user (MMSE MUD) is presented. The investigations are carried out by examining the relationship between MMSE combining (MMSEC) detection technique and MMSE MUD technique. Simulation results show that in a full-load system, both MMSEC and MMSE-MUD technique have the same performance, while in a non full-load system, MMSE-MUD technique outperforms its MMSEC counterpart.

Tóm tắt. Trong bài báo này chúng tôi phân tích và đánh giá một số kỹ thuật tách tín hiệu cho kênh đường xuống của hệ thống CDMA đa sóng mang thông qua chỉ tiêu chất lượng tỉ số lỗi bít (BER). Đồng thời chúng tôi cũng nghiên cứu chi tiết một giải pháp tách tuyến tính, đó là kỹ thuật tách tín hiệu đa người dùng dựa trên lỗi bình phương trung bình tối thiểu (MMSE) trên từng người dùng (MMSE MUD). Nghiên cứu được tiến hành thông qua phân tích sự tương quan giữa phương pháp này và kỹ thuật tách tín hiệu kết hợp theo thuật toán MMSE (MMSEC). Các kết quả mô phỏng cho thấy đối với hệ thống đủ người dùng, cả hai kỹ thuật này đều có chỉ tiêu chất lượng như nhau. Nhưng khi hệ thống có số người dùng bất kỳ thì kỹ thuật tách tín hiệu theo thuật toán MMSE MUD đạt được chất lượng tốt hơn.
\end{abstract}

\section{INTRODUCTION}

Multi-carrier Code Division Multiple Access (MC-CDMA) is a combination of the multicarrier transmission technique known as Orthogonal Frequency Multiplexing (OFDM) and Code Division Multiple Access (CDMA). Since 1993 Multi-carrier CDMA has drawn great interest of researchers. It has been shown to be a good means of supporting multimedia services in the future mobile radio communication $([1-5])$. The OFDM modulation is robust against multipath and ensures frequency spectrum utilization efficiency. The CDMA allows simultaneous communications between different transceivers by allocating to each transmission link a spreading sequence that has good orthogonal properties with spreading sequences of other users. Instead of spreading the binary information in the time domain as MC-DSCDMA ([6]), the MC-CDMA spreading is performed in the frequency domain. In other words, a fraction of the symbol corresponding to a chip of the spreading code is transmitted through 
a different sub-carrier.

For multi-carrier transmission, it is essential to have frequency non-selective fading over each sub-carrier. Furthermore, in synchronous MC-CDMA system such as the forward link mobile radio communication channel, orthogonal spreading codes, such as the Walsh-Hadamard codes, are often desirable because they make multiple access interference (MAI) be totally eliminated in a Gaussian channel. However, when a signal is transmitted through a multipath channel, if the coherent bandwidth $(\Delta f)_{c}$ of the channel is small compared with the bandwidth of the transmitted signal, a frequency selective fading will appear. Consequently, all the sub-carrier have different amplitude levels and different phase shifts, which results in a loss of orthogonality among users, thereby generating MAI and reducing the system performance.

Several detection techniques have been proposed to mitigate the MAI degradation. The Single User Detection (SUD) techniques perform a single tap equalization per sub-channel to compensate for the phase and amplitude distortion caused by the frequency selective fading channel. Various basic SUD techniques such as Maximum Ratio Combining (MRC), Equal Gain Combining (EGC), Orthogonal Restoring Combining (ORC) and MMSEC have been presented in [7-8]. Besides, the comparison of different equalization strategies is also demonstrated in [9]. All the SUD techniques require low computational complexity. Among those techniques, MMSEC technique, which is applied independently to each sub-carrier ([10]), achieves the best performance. However, the SUD techniques usually do not take into account any information about the MAI, for instance: the other users spreading codes and relative signal power of each user in system. Therefore, they usually limit the performance of system.

Consequently, some multi-users detection (MUD) techniques have been devised to improve system performance by explicitly considering signals of other subscribers for the signal detection of a specific user [11]. The MUD techniques utilizing the non-linear solution based on Interference Cancellation have been considered in [13-14]. In order to reduce MAI, linear MMSE detection techniques in $[2,15]$ minimize the mean square error at the input of the threshold detector (after the despreading process).

In this paper, we use MC-CDMA receiver based on MMSE criterion applied per user to reduce the multi-user interference (MUI). This technique requires the spreading codes of different users to be known at the receiver. In addition, it is an optimal technique for any number of active users under the assumption that all the users have the same power. The comparison between MMSEC detection and MMSE MUD per user detection in the case of downlink is proved for full load and especially even for non full load systems.

The organization of this paper is as follow. In Section 2, a description of the MC-CDMA transmission scheme is given. The various frequency equalization techniques for single user detection, the algorithms on linear MMSE MUD per user for multiuser detection as well as the relationship between MMSEC detection and MMSE MUD are derived in Section 3. Next, in Section 4, we show the simulation results, which evaluate the BER performance of MC-CDMA systems with different equalizations, and compare the MMSEC technique with MMSE MUD technique. Finally, Section 5 presents concluding remarks.

\section{MC-CDMA SYSTEM DESCRIPTION}

The block diagram of the considered MC-CDMA transmitter and receiver is depicted in 
Figure 1. We consider the forward link MC-CDMA system of $K$ independent data streams corresponding to $K$ different users. Data symbol $d_{(k)}(i)$ at the time $i$ is assigned to user $k(k=1,2, \ldots K), \boldsymbol{d}=\left[d_{(1)}(i), \ldots, d_{(K)}(i)\right]^{T}$ is a $K \times 1$ vector containing the data symbol of each user, where [.] denotes matrix transposition. The data symbol $d_{(k)}(i)$ is multiplied with its user specific spreading code $\boldsymbol{c}_{k}=\left[c_{k, 1}, c_{k, 2}, \ldots, c_{k, L}\right]^{T}$ of length $L$. We assume that the spreading codes are mutually orthogonal. Here, $L$ corresponds to the bandwidth expansion factor and is equal to the maximum number of simultaneous active users (with full load system). Orthogonal Walsh-Hadamard (WH) codes are used for spreading. For simplicity, we consider a spreading factor equal to the number of sub-carriers $N_{c}$ and $C$ is a $N_{c} \times K$ matrix of all users given by

$$
\boldsymbol{C}=\left[\begin{array}{cccc}
c_{1,1} & c_{2,1} & \cdots & c_{K, 1} \\
c_{1,2} & c_{2,2} & \cdots & c_{K, 2} \\
\vdots & \vdots & \vdots & \vdots \\
c_{1, N_{c}} & c_{2, N_{c}} & \cdots & c_{K, N_{c}}
\end{array}\right]
$$

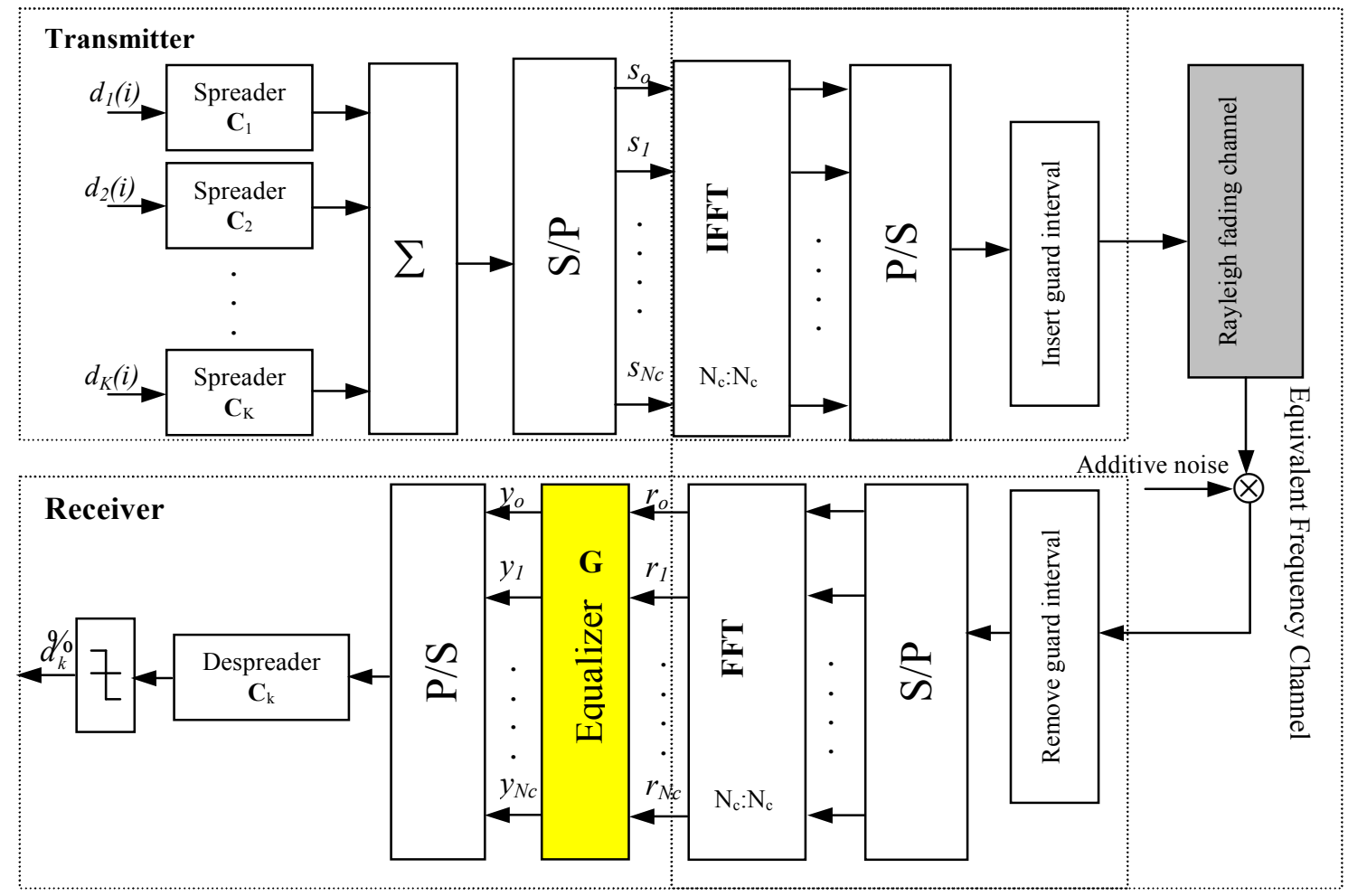

Figure 1. MC-CDMA transmitter and receiver for down link

Here, the columns of $\boldsymbol{C}$ matrix are the spreading sequences of active users. As we consider the synchronous downlink of the MC-CDMA system, the different data modulated spreading codes of the $K$ users can be added before the Serial-to-Parallel (S/P) conversion. Moreover, the $K$ user signals are assumed to be transmitted with the equal powers. Data symbols of each user are QPSK modulated. The transmitted signal of MC-CDMA system in downlink can be given as 


$$
S=C d
$$

where $\boldsymbol{S}=\left[s_{1}, s_{2}, \ldots, s_{N_{c}}\right]^{T}$ is a $N_{c} \times 1$ vector containing the transmitted data symbol per sub-carrier. In this investigations, frequency non-selective Rayleigh fading per sub-carrier and time invariance during on OFDM symbol are assumed. The absence of Inter-Symbol Interference (ISI) and Inter-Carrier Interference (ICI) is also guaranteed by the use of an appropriate guard interval, which is cyclic prefix added to each OFDM symbol ([16]). The complex channel fading coefficients are supposed to be independent for each sub-carrier and constant during the transmission of each OFDM symbol. Under the above assumptions, the IFFT and guard interval insertion block, the Rayleigh fading channel along with the FFT and guard interval removal block are considered as an equivalent frequency channel, as illustrated in Figure 1.

At the receiver the guard interval is removed and the inverse OFDM operation is performed. Then, the received signals can be expressed as

$$
\boldsymbol{r}=\left[r_{1}, r_{2}, \ldots, r_{N_{c}}\right]^{T}=\boldsymbol{H} \boldsymbol{S}+\boldsymbol{N}=\boldsymbol{H} \boldsymbol{C d}+\boldsymbol{N}
$$

where $\boldsymbol{H}$ is a $N_{c} \times N_{c}$ diagonal matrix containing the complex channel attenuation of each sub-carrier

$$
\boldsymbol{H}=\left[\begin{array}{cccc}
H_{1} & 0 & \cdots & 0 \\
0 & H_{2} & \cdots & 0 \\
\vdots & \vdots & \vdots & \vdots \\
0 & 0 & \cdots & H_{N_{c}}
\end{array}\right]
$$

and $\boldsymbol{N}=\left[N_{1}, N_{2}, \ldots, N_{N_{c}}\right]^{T}$ is $N_{c} \times 1$ vector of Additive White Gaussian Noise (AWGN) components with $N_{n}$ representing the noise at the sub-carrier $n^{\text {th }}$ which has variance $\sigma_{n}^{2}=$ $E\left[\left|N_{n}\right|^{2}\right], n=1,2, \ldots, N_{c}$.

\section{EQUALIZATION TECHNIQUES}

Detectors for MC-CDMA can be classified into the two basic categories: Single User Detection and Multi-User Detection.

\subsection{Single user detection (SUD)}

In the first category, the receiver has knowledge of spreading code employed by the user of interest only, which means that it has no knowledge of the spreading codes employed by other users. Interference from other users is assimilated to additive channel noise and no attempt is made to compensate for it. In SISO MC-CDMA mobile radio system, SUD is realized by one tap equalization to compensate for the distortion due to fading on each sub-carrier, followed by using specific despreading.

After equalization the receiver signal can be written as

$$
\boldsymbol{Y}=\left[y_{1}, y_{2}, \ldots, y_{N_{c}}\right]^{T}=\boldsymbol{G r}=\boldsymbol{G H} \boldsymbol{C d}+\boldsymbol{G N}
$$

The estimated symbol of the $k^{\text {th }}$ user is equal to:

$$
Q\left(\widetilde{d}_{k}\right)=Q\left(\boldsymbol{c}_{k, n}^{*} G_{n} r_{n}\right)
$$


where $Q($.$) denotes quantization operation. The N_{c} \times N_{c}$ matrix $\boldsymbol{G}$ contains complex equalization coefficients obtained from channel estimation, which can be known through transmitted pilot symbols inserted between the OFDM signals.

In the sequel, we will describe different basic equalization techniques of this category in details.

\subsubsection{Maximum Ratio Combining (MRC)}

This technique corrects the phase shift by multiplying the receiver signal with the conjugate complex channel coefficient

$$
G_{n}=H_{n}^{*}
$$

where $(.)^{*}$ denotes complex conjugation, and $H_{n}\left(n=1,2, \ldots, N_{c}\right)$ are the diagonal components of $\boldsymbol{H}$. The drawback of MRC in the downlink of SISO MC-CDMA system is that it destroys the orthogonality between spreading codes and thus, additionally enhances the multiple access interference $([12])$.

\subsubsection{Equal Gain Combining (EGC)}

EGC, also called phase equalization, compensates only for the phase rotation caused by the channel by choosing the equalization coefficient as

$$
G_{n}=\frac{H_{n}^{*}}{\left|H_{n}\right|}
$$

EGC is the simplest single user detection techniques, since it only needs information about the phase of the channel.

\subsubsection{Orthogonal Restoring Combining (ORC)}

ORC inverses the channel transfer function and can eliminate multiple access interference by restoring the orthogonality between the users with an equalization coefficient chosen as

$$
G_{n}=\frac{1}{H_{n}}
$$

In the literature, ORC is also called Zeros-Forcing (ZF). The drawback of ZF equalization is that for small amplitudes of $H_{n}$ the equalizer enhances noise $N_{n}$ in such a way that the signal to noise ratios $\gamma_{c}$ (the average SNR per carrier at the input of the data detector $\gamma_{c}=$ $\left.\frac{\boldsymbol{E}\left[\left|s_{n}\right|^{2}\right]}{\sigma_{n}^{2}}=\frac{E_{\text {carrier }}}{\sigma_{n}^{2}}\right)$ may reduce to zero on some sub-carriers.

\subsubsection{Minimum mean square error combining (MMSEC)}

Equalization according to the MMSE criterion minimizes the mean square value of the error $\varepsilon_{n}$ between the signal $S_{n}$ transmitted on sub-carrier $n^{\text {th }}$ and the assigned output $y_{n}$ of the equalizer.

$$
\varepsilon_{n}=s_{n}-G_{n} r_{n}
$$

The mean square error is

$$
G_{n}=\min _{G_{n}} E\left[\left|\varepsilon_{n}\right|^{2}\right]
$$

The receiver signal at $n^{\text {th }}$ sub-carrier is: 


$$
r_{n}=\sum_{k=1}^{K} H_{n} c_{k, n} d_{k}+N_{n}=H_{n} s_{n}+N_{n}
$$

where $s_{n}=\sum_{k=1}^{K} c_{k, n} d_{k}$ and $\hat{s}_{n}=y_{n}=G_{n} r_{n}$ are the transmitted signal on $n^{\text {th }}$ sub-carrier and receiver signal after equalizer, respectively. According to the Wiener - Hopf equation, the equalization coefficient matrix $G_{n}$ is equal to

$$
G_{n}=R_{n}^{-1} p_{n}
$$

where $R_{n}^{-1}$ is the autocorrelation of the received signal $r_{n}$ and $p_{n}$ is the cross-correlation signal between the desired signal on $n^{t h}$ sub-carrier $s_{n}$ and the received signal $r_{n}$.

$$
\begin{aligned}
R_{n} & =\boldsymbol{E}\left[r_{n} r_{n}^{*}\right]=\boldsymbol{E}\left[\left(\sum_{k=1}^{K} H_{n} c_{k, n} d_{k}+N_{n}\right)\left(\sum_{k=1}^{K} H_{n}^{*} c_{k, n} d_{k}^{*}+N_{n}^{*}\right)\right]=\sum_{k=1}^{K} E_{\text {chip }}\left|H_{n}\right|^{2}+\sigma_{n}^{2} \\
& =K E_{\text {chip }}\left|H_{n}\right|^{2}+\sigma_{n}^{2}=E_{\text {carrier }}\left|H_{n}\right|^{2}+\sigma_{n}^{2}=E_{\text {carrier }}\left(\left|H_{n}\right|^{2}+\frac{1}{\gamma_{c}}\right)
\end{aligned}
$$

or

$$
\begin{gathered}
R_{n}=\frac{K}{L} E_{b}\left|H_{n}\right|^{2}+\sigma_{n}^{2} \\
p_{n}=\boldsymbol{E}\left[r_{n}^{*} s_{n}\right]=\boldsymbol{E}\left[\left(\sum_{k=1}^{K} H_{n} c_{k, n} d_{k}+N_{n}\right)^{*}\left(\sum_{k=1}^{K} c_{k, n} d_{k}\right)\right]=K E_{\text {chip }} H_{n}^{*}=E_{c a r r i e r} H_{n}^{*} \\
p_{n}=E_{\text {carrier }} H_{n}^{*}
\end{gathered}
$$

where $\left|c_{k, n}\right|^{2}=\frac{1}{L} ; \boldsymbol{E}\left[\left|d_{k} d_{k}^{*}\right|^{2}\right]=E_{b} ; E_{\text {carrier }}, E_{c h i p}$ and $E_{b}$ are the energy per sub-carrier; the energy per chip and the energy per symbol before spreading, respectively, the relation between them is given by

$$
E_{\text {chip }}=\frac{1}{L} E_{b}, \text { and } E_{\text {carrier }}=K E_{\text {chip }}=\frac{K}{L} E_{b}
$$

The equalization coefficient based on MMSE criterion applied independently per carrier are given as

$$
G_{n}=R_{n}^{-1} p_{n}=\frac{E_{\text {carrier }} H_{n}^{*}}{E_{\text {carrier }}\left(\left|H_{n}\right|^{2}+\frac{1}{\gamma_{c}}\right)}=\frac{H_{n}^{*}}{\left(\left|H_{n}\right|^{2}+\frac{1}{\gamma_{c}}\right)}
$$

where $\gamma_{c}$ is the SNR per sub-carrier and relates to the SNR per symbol $\gamma_{b}=\frac{K \gamma_{c}}{L}$. Then the optimal coefficients of the equalizer are equal to

$$
G_{n}=\frac{H_{n}^{*}}{\left|H_{n}\right|^{2}+\frac{L}{K} \frac{1}{\gamma_{b}}}
$$

When the system has only one user, the equalization coefficient matrix $G_{n}$ is equal to

$$
G_{n}=\frac{H_{n}^{*}}{\left|H_{n}\right|^{2}+\frac{L}{\gamma_{b}}}=\frac{H_{n}^{*}}{\left|H_{n}\right|^{2}+\frac{1}{\gamma_{c h i p}}}
$$


and when the system is full load $(K=L)$, the formula (19) becomes the formula (18). And then the estimated data symbol of the user $k^{\text {th }}$ is

$$
\widetilde{d}_{k}=\boldsymbol{c}_{k, n}^{*} G_{n} r_{n}=\sum_{n=1}^{N_{c}} \frac{\boldsymbol{c}_{k, n}^{*} H_{n}^{*}}{\left|H_{n}\right|^{2}+\frac{L}{K} \frac{1}{\gamma_{b}}} r_{n}
$$

The MMSEC equalization corrects the phase shift and the attenuation of the channel fading, taking into account the number of active users $K$ and the present signal to noise ratio.

For all these basic techniques, the matrix $\boldsymbol{G}$ is diagonal and the receiver sequence is equalized by using a bank of $N_{c}$ adaptive one tap equalizers. This means that the complexity of the equalizer is low. Among all the SUD techniques, the MMSEC equalization per sub-carrier can offers the best results. However, MMSEC equalization per carrier method is still not optimal because it does not take into account the despreading process and thus does not minimize the mean square error at the input of the threshold detector. Thus, to this end, we analyze an improved method base on the linear MMSE per user (MMSE MUD) technique to detect multi-user interference.

\subsection{Multi-user detection (MUD)}

In this section we introduce Minimum Mean Square Error MUD equalization method which belongs to the second category of MC-CDMA detector. The basic idea of MMSE MUD is to minimize the mean square error between transmitted data symbol $d_{k}$ and the estimated data symbol $\hat{d}_{k}$.

$$
\hat{d}_{k}=\boldsymbol{W}_{k}^{H} \boldsymbol{r}
$$

where $\boldsymbol{W}_{k}=\left[w_{k}^{1}, w_{k}^{2}, \ldots, w_{k}^{N_{c}}\right]^{T}$ is the optimal weighting vector. We have

$$
\min _{\boldsymbol{W}_{k}} \boldsymbol{E}\left[\left|d_{k}-\boldsymbol{W}_{k}^{H} \boldsymbol{r}\right|^{2}\right]
$$

Applying to the Wiener-Hopf equation, the optimal weighting vector is equal to

$$
W_{k}=R_{r r}{ }^{-} 1 p_{r d}
$$

where $\boldsymbol{R}_{\boldsymbol{r} \boldsymbol{r}}$ is the autocorrelation matrix of the received vector $\boldsymbol{r}$ and $\boldsymbol{p}_{\boldsymbol{r} \boldsymbol{d}}$ is the cross-correlation vector between the desired symbol, $d_{k}$ and the receiver vector $\boldsymbol{r} . \boldsymbol{R}_{\boldsymbol{r} \boldsymbol{r}}$ is given by

$$
\begin{gathered}
\boldsymbol{R}_{\boldsymbol{r r}}=\boldsymbol{E}\left[\boldsymbol{r} \boldsymbol{r}^{\boldsymbol{H}}\right]=\boldsymbol{E}\left[(\boldsymbol{H} \boldsymbol{C} \boldsymbol{d}+\boldsymbol{N})(\boldsymbol{H} \boldsymbol{C} \boldsymbol{d}+\boldsymbol{N})^{H}\right]=\boldsymbol{E}\left[\boldsymbol{H} \boldsymbol{C} \boldsymbol{d} \boldsymbol{d}^{\boldsymbol{H}} \boldsymbol{C}^{\boldsymbol{H}} \boldsymbol{H}^{\boldsymbol{H}}\right]+\boldsymbol{E}\left[\boldsymbol{N} \boldsymbol{N}^{H}\right] \\
=\boldsymbol{H} \boldsymbol{C} \boldsymbol{E}\left[\boldsymbol{d} \boldsymbol{d}^{H}\right] \boldsymbol{C}^{H} \boldsymbol{H}^{H}+\sigma_{n}^{2} \boldsymbol{I}_{N_{c} \times N_{c}} \\
\boldsymbol{p}_{\boldsymbol{r} \boldsymbol{d}}=\boldsymbol{E}\left[d_{k}^{*} \boldsymbol{r}\right]=\boldsymbol{E}\left[d_{k}^{*}(\boldsymbol{H} \boldsymbol{C} \boldsymbol{d}+\boldsymbol{N})\right]=\boldsymbol{H} \boldsymbol{C} E\left[d_{k}^{*} \boldsymbol{d}\right]=\boldsymbol{E}\left[\left|d_{k}\right|^{2}\right] \boldsymbol{H} \boldsymbol{C}_{k} \\
\boldsymbol{p}_{\boldsymbol{r d}}=\boldsymbol{E}\left[\left|d_{k}\right|^{2}\right]\left[\begin{array}{cccc}
H_{1} & 0 & \cdots & 0 \\
0 & H_{2} & \cdots & 0 \\
\vdots & \vdots & \vdots & \vdots \\
0 & 0 & \cdots & H_{N}
\end{array}\right]\left[\begin{array}{c}
c_{k, 1} \\
c_{k, 2} \\
\vdots \\
c_{k, N_{c}}
\end{array}\right]=\boldsymbol{E}\left[\left|d_{k}\right|^{2}\right]\left[\begin{array}{c}
c_{k, 1} H_{1} \\
c_{k, 2} H_{2} \\
\vdots \\
c_{k, N_{c}} H_{N_{c}}
\end{array}\right]
\end{gathered}
$$

Since the user signals have the same power and are independent, we can have $\boldsymbol{E}\left[\left|d_{k}\right|^{2}\right]=E_{b}$ and $\boldsymbol{E}\left[\boldsymbol{d} \boldsymbol{d}^{H}\right]=E_{b} \boldsymbol{U}$, where $\boldsymbol{U}=\left\{u_{i k}\right\}$ is the diagonal matrix with the term $u_{k k}=1$, if the user $k$ is active, and $u_{k k}=0$ if the user $k$ is inactive. 


$$
\boldsymbol{W}_{k}^{H}=\boldsymbol{H}^{H} \boldsymbol{C}_{k}^{H}\left(\boldsymbol{H} \boldsymbol{C} \boldsymbol{U} \boldsymbol{C}^{H} \boldsymbol{H}^{H}+\frac{\sigma_{n}^{2}}{E_{b}} \boldsymbol{I}_{N_{c} \times N_{c}}\right)^{-1}
$$

The optimal weighting vector can be expressed as

$$
\boldsymbol{W}_{k}^{H}=\boldsymbol{C}_{k}^{H} \boldsymbol{G}
$$

Hence, the equalization coefficient matrix of the MMSE MUD per user is equal to:

$$
\boldsymbol{G}=\boldsymbol{H}^{H}\left(\boldsymbol{H} \boldsymbol{C} \boldsymbol{C}^{H} \boldsymbol{H}^{H}+\frac{\sigma_{n}^{2}}{E_{b}} \boldsymbol{I}_{N_{c} \times N_{c}}\right)^{-1}
$$

When the system is full load $(K=L)$, the quantity $\boldsymbol{C} \boldsymbol{U} \boldsymbol{C}^{H}$ is equal to the identity matrix and the equalization coefficient matrix $\boldsymbol{G}$ is a diagonal matrix with the $n^{\text {th }}$ sub-carrier equalization coefficient is calculated by equation (18). On the other hand, in the non full load case $(K<L)$, the equalization coefficient matrix $\boldsymbol{G}$ is no longer diagonal.

In the special case, with $K=1$, the $\boldsymbol{U}$ is given by $\mathbf{1 1 ^ { T }}$, where $\mathbf{1}$ is a L-by- 1 vector with all the entries equal to one, and the autocorrelation matrix $\boldsymbol{R}_{\boldsymbol{r} r}$ becomes

$$
\boldsymbol{R}_{\boldsymbol{r} \boldsymbol{r}}=E_{b} \boldsymbol{H} \boldsymbol{C}_{k} \mathbf{1 1}^{T} \boldsymbol{C}_{k}^{H} \boldsymbol{H}^{H}+\sigma_{n}^{2} \boldsymbol{I}_{N_{e} \times N_{e}}
$$

The cross-correlation vector is

$$
\boldsymbol{p}_{k}=E_{b} \boldsymbol{H} \boldsymbol{C}_{k} \mathbf{1}
$$

Defining $\boldsymbol{x}_{k}=\boldsymbol{H} \boldsymbol{C}_{k} \mathbf{1}$ and using matrix inversion lemma, the optimal weighting vector is equal to

$\boldsymbol{W}_{k}=\boldsymbol{R}_{k}^{-1} \boldsymbol{p}_{k}=\frac{E_{b}}{\sigma_{n}^{2}}\left(\boldsymbol{I}-\frac{\gamma_{b}}{1+\gamma_{b}\left\|\boldsymbol{x}_{k}\right\|^{2}} \boldsymbol{x}_{k} \boldsymbol{x}_{k}^{H}\right) \boldsymbol{x}_{k}=\frac{E_{b}}{\sigma_{n}^{2}}\left(\boldsymbol{x}_{k}-\frac{\gamma_{b}\left\|\boldsymbol{x}_{k}\right\|^{2}}{1+\gamma_{b}\left\|\boldsymbol{x}_{k}\right\|^{2}} \boldsymbol{x}_{k}\right)=\left(\frac{\gamma_{b}}{1+\gamma_{b}\left\|\boldsymbol{x}_{k}\right\|^{2}}\right) \boldsymbol{x}_{k}$

Then the optimal weighting vector can be expressed as

$$
\boldsymbol{W}_{k, n}^{H}=\frac{\gamma_{b} \boldsymbol{c}_{k, n}^{*} H_{n}^{*}}{1+\gamma_{b} \sum_{l=1}^{L}\left|H_{n}\right|^{2}\left|\boldsymbol{c}_{k, n}\right|^{2}}=\frac{\boldsymbol{c}_{k, n}^{*} H_{n}^{*}}{\frac{1}{L} \sum_{l=1}^{L}\left|H_{n}\right|^{2} \mid+\frac{1}{\gamma_{b}}}
$$

Comparing equations (32) and (20), we observe that there is difference between the equalization coefficient of the MMSEC technique and that of the MMSE MUD one. As confirmed by simulation results due to that difference, the MMSE MUD offers better BER performance than the MMSEC when the system has only one active user.

From equation (27), if we want to implement the MMSE MUD algorithm we must know $\boldsymbol{H}$ and $\boldsymbol{U}$. Furthermore, the inversion of $\boldsymbol{H} \boldsymbol{C} \boldsymbol{U} \boldsymbol{C}^{H} \boldsymbol{H}^{H}+\frac{\sigma_{n}^{2}}{E_{b}} \boldsymbol{I}_{N_{c} \times N_{c}}$ matrix may be a time consuming operation, particularly for large length $L$ of codes.

\section{SIMULATION RESULTS}

\subsection{Performance comparison of the detection techniques}

The following results are obtained using Monte Carlo simulations Matlab code. In Figure 2, 3 and 4 the graph of BER versus SNR in dB of the MC-CDMA system of MRC, EGC, ORC and MMSEC equalizations with different number of active users are shown. The simulations 
were performed without channel coding and interleaving. Each of the independent sub-carrier is QPSK modulated at the transmitter and then multiplied by an uncorrelated Rayleigh fading. We assume that the estimation of the frequency channel response for each sub-carrier is correct and the channel matrix $\boldsymbol{H}$ is perfectly known to the receiver, and therefore, it is possible to calculate the optimum weights by a direct matrix inversion operation.

Figure 2 shows the performance of a full-load system where the number of active users is equal to the length of the WH codes, $K=L=64$, (maximum user capacity). Without using an equalizer, the performance of the system is very bad. Even in the case MRC equalizer is presented, the performance is still not good enough for practical use. The loss of orthogonality of the $\mathrm{WH}$ codes is heightened in the receiver when applying MRC. The ORC, zero-forcing equalization restores the orthogonality between the user signals and avoids MAI. However, it introduces noise application which is especially high at low SNRs. EGC avoids noise application but does not counteract the MAI caused by the loss of the orthogonality between the user signals, resulting in a high error floor. The single user detection based on minimum mean square error per carrier equalization offers good results. The matched filter (MF) bound is also given for reference. The MMSEC technique outperforms the other techniques because it avoids an excessive noise application for low signal to noise ratios, while keeping the orthogonality among users for large SNRs.

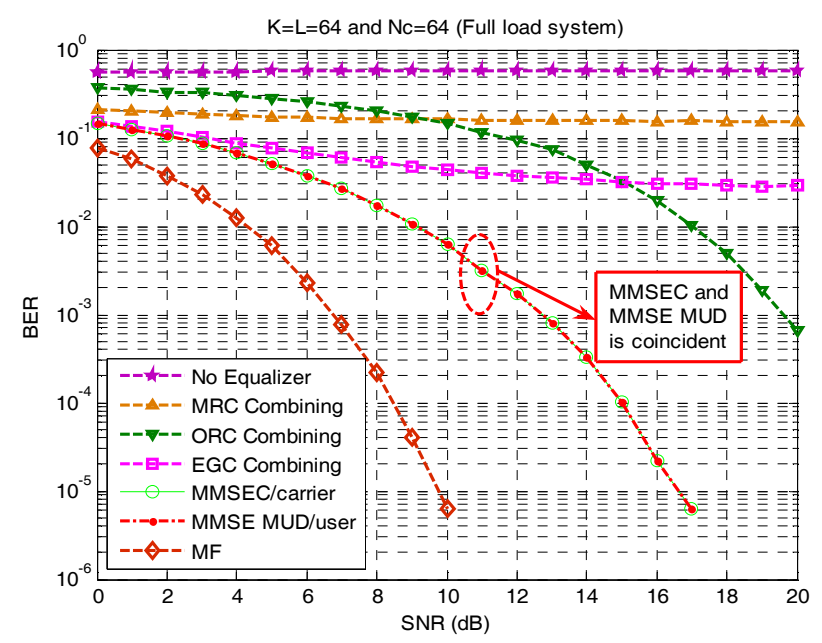

Figure 2. Different detection performance for $K=L=N_{c}=64$ (full load system) in the MC-CDMA

\subsection{The relationship between MMSE MUD per user and MMSEC per sub-carrier}

In Figure 2, the system is full-loaded $(K=L)$, the $K$ user signals are supposed to be transmitted with the same power $E\left[\left|d_{1}\right|^{2}\right]=\cdots=E\left[\left|d_{K}\right|^{2}\right]=E_{b}$, then the algorithm of the MMSE MUD per user contains the quantity $\boldsymbol{C} \boldsymbol{U} \boldsymbol{C}^{H}$ which will be equal to the identity matrix. Thus, equation (29) is similar to equation (18) and the equalization coefficient matrix $\boldsymbol{G}$ is a diagonal matrix with the $n^{\text {th }}$ sub-carrier equalization coefficient being calculated by equation (18). In that case, the performance of the two MMSE approaches are the same and the curve of the MMSE MUD coincident with the curve of MMSEC detection technique (see Figure 2).

On the other hand, in the Figure 3 and Figure 4, for the non-full loaded systems $(K<L)$, 
the equalization coefficient matrix $G$ is not a diagonal matrix. Therefore, the equalization coefficient matrix $\boldsymbol{G}$ of the MMSE MUD obtained from equation (29) outperforms the algorithm MMSEC per sub-carrier based on equation (18). This preeminent advantage can be observed in Figure 3 and Figure 4 with the case $K=56$ and $K=32$ (corresponding to a system load equal to $88 \%$ and $50 \%$ respectively).

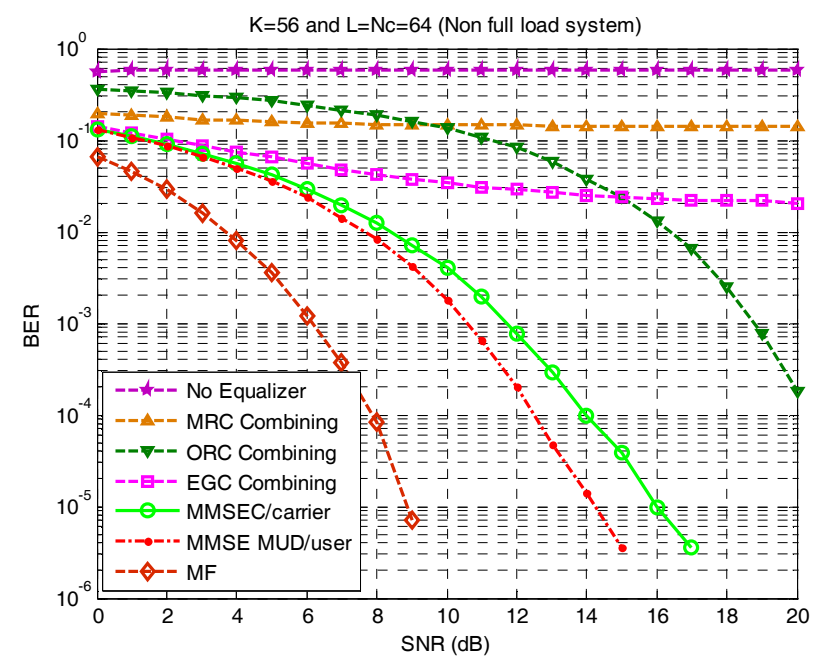

Figure 3. Different detection performance for $K=56<L=N_{c}=64$ (Non full load system)

Those results are logical because MMSE MUD algorithm minimizes the decision error by taking into account the despreading process instead of minimizing the error independently on each sub-carrier, thus both the interference and the noise enhancement are minimized.

Furthermore, it allows adjusting the coefficients using decisions on the information symbols assuming that those decisions are correct.

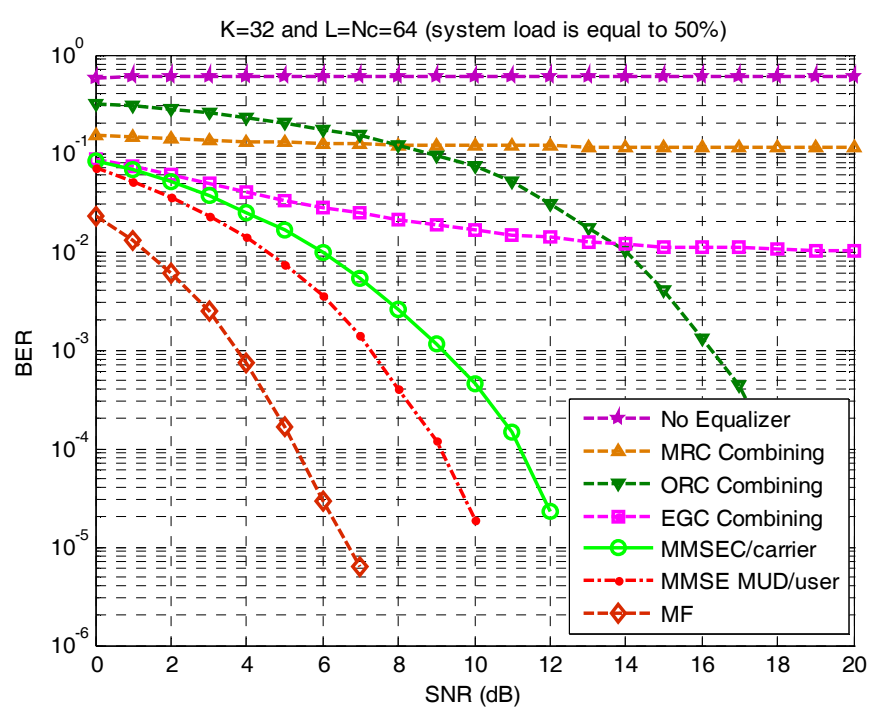

Figure 4. Different detection performance for $K=32<L=N_{c}=64$

(system load equal to 50\%) 
In order to compare the performances of MMSEC and MMSE MUD in more details, we range the number of active users from 1 to 64 then estimate the required SNR to achieved a $B E R=10^{-3}$. The relationship between the number of active users and the required SNR is shown on Figure 5. Again, the Zeros-Forcing technique, which is better than MRC and EGC, is outperformed by MMSEC and MMSE MUD. The difference between MMSEC and MMSE MUD can be easily observed in this figure. In the case the number of active users is full-loaded, both equalization techniques give the identical performance and the user/SNR curves meet each other at a point. On the other hand, when the system is non-full loaded, the MMSE MUD based on the MMSE per user criterion achieves a gain of more than $2 \mathrm{~dB}$ with $K=32$ which corresponds to system equal to $50 \%$. Particularly, when the system has one active user, the MMSE MUD still performs better than the MMSEC, as illustrated in Figure 5.

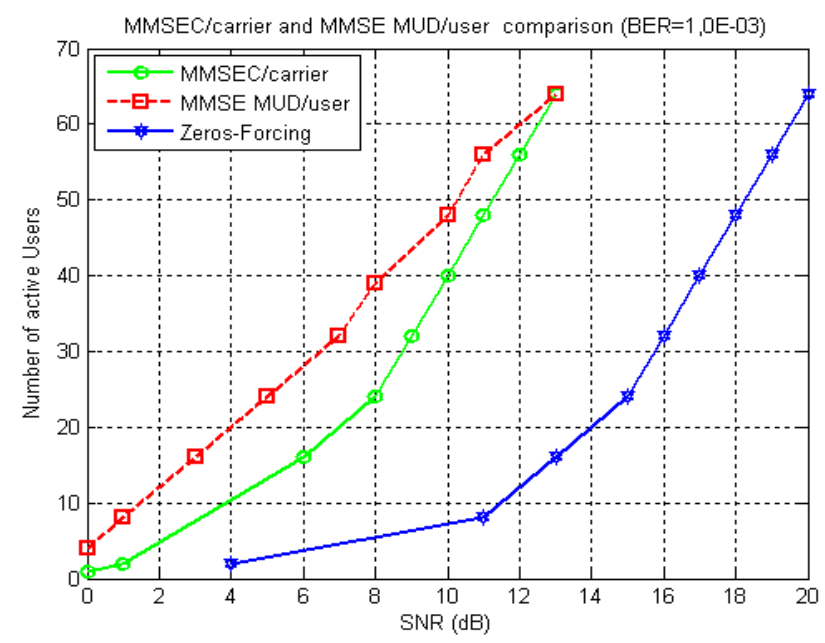

Figure 5. Comparison of the number $K$ of active users between MMSEC and MMSE MUD versus SNR with $B E R=10^{-3}, L=N_{c}=64$

\section{CONCLUSIONS}

The bit error rate performances of single user detection and multi-user detection techniques for the downlink of a MC-CDMA system are presented in this paper. Also, the relationship between MMSEC and MMSE MUD detection are compared and evaluated. It was seen that the MMSE MUD outperforms all other detection techniques, especially for high bit rate scenarios, whereas the MRC, EGC, ZF detections result in very poor performances. The MMSE MUD per user approach offers for non-full load systems a significant gain compared to the MMSEC per carrier technique. In particular for $L=32$ (50\% of the system), the MMSE MUD per user criterion achieves a gain of more than $2 \mathrm{~dB}$ in comparison with MMSEC. For a MC-CDMA system, the probability that the maximum number $\mathrm{K}$ of active users are working at the same time is usually small. Hence, the MMSE MUD is a good choice for non-full load system. However, the MMSE MUD per user is computationally excessive. It was also observed that the MMSEC could provide a better trade-off between performance and complexity, especially under high load conditions. 


\section{REFERENCES}

[1] N. Yee, J. P. Linnartz, G. Fettweis, Multi-Carrier CDMA in indoor wireless radio networks, PIMRC '93 vol.1, Yokohama, Japan, Sept.1993 (109-113).

[2] A. Chouly, A. Brajal, S. Jourdan, Orthogonal multicarrier techniques applied to direct sequence spread spectrum CDMA systems, GLOBECOM'93, Houston, USA, Nov. 1993 (1723-1728).

[3] K. Fazel, L. Papke, On the performance of convolutionally coded CDMA/OFDM for mobile communication system, PIMRC'93 Sept. 1993 (468-472).

[4] V. M. DaSilva and F. S. Sousa, Multicarrier orthogonal signals for quasi-synchronous communications systems, IEE J. Sel.Areas Commun. 12 (5) June 1994 (842-852).

[5] F. A. Sourour and Nakagawa, Performance of orthogonal multicarrier CDMA in a multipath fading channel, IEEE Trans. Commun, 44 (3) 1996 (356-367).

[6] S. Kondo and L. B. Milstein, Performance of multicarrier DS CDMA system, IEEE Trans. On Commun. 44 (2) Feb. 1996 (238-246).

[7] S. Hara, R. Prasad, Overview of multicarrier CDMA, IEEE Communications Magazine 35 Dec. 1997 (126-133).

[8] R. L. Gouable and M. Helard, Performance of MC-CDMA systems in multipath indoor enviroments coparison with COFDM-TDMA system, 3G Mobile Communication Technologies, first International Conference, IEE Conf. Publ. (471) March 2000 (81-85).

[9] M. A. Cooper, S. M. D. Armour, Y. Q. Bian, and J. P. McGeehan, Comparison of equalisation strategies for multi-carrier CDMA, "International Conference on Consumer Electronics", 2003 (298-299).

[10] S. Kaisei, Analytical performance evaluation of OFDM-CDMA mobile radio systems, Proc. First European Personal and Mobile Communications Conf. (EPMCC'95) Bologna, Italy, Nov. 1995 (215-220).

[11] D. Mottier, D. Castelain, J. F. Helard, and J. Y Baudais, Optimum and sub-optimum linear MMSE multi-user-detection for multicarrier CDMA transmission systems, Vehicular Technology Conference 2001 VTC'2001 2 Oct. 2001 (868-872).

[12] S. Kaiser, "Multi-Carrier CDMA Radio Systems - Analysis and Optimization of Detection, Decoding, and Channel Estimation", PhD. Thesis, VDI-Verlag, Fortschrittberichte VDI, Series 10, No. 531, 1998.

[13] N. Benvenuto, P. Bisaglia, Parallel and successive interference cancellation for MC-CDMA and their near-far resistance, Vehicular Technology Conference 2003 VTC'2003 2 Oct. 2003 (1045-1049).

[14] H. Wang, Z. Li, J. Lilleberg, Equalized parallel interference cancellation for MC-CDMA multicode downlink transmission, Wireless Communication and Networking Conference 2004 WCNC'04 3 March 2004 (1812-1816).

[15] N. Yee, J. P. Linnartz, Wiener filtering of multi-carrier CDMA in a rayleigh fading channel, PIMRC'94 4 (1344-1347).

[16] S. Hara and R. Prasad, "Multicarrier techniques for 4G mobile communications", Artech House, 2003.

Received on August 1 - 2006 